\title{
Contribution of audiovestibular tests to the topographic diagnosis of sudden deafness
}

\author{
Jeanne Oiticica ${ }^{1}$, Roseli Saraiva Moreira Bittar ${ }^{2}$, Claudio Campi de Castro ${ }^{3}$, Signe Grasel ${ }^{4}$, Larissa Vilela Pereira ${ }^{5}$, \\ Sandra Lira Bastos ${ }^{5}$, Alice Carolina Mataruco Ramos ${ }^{5}$, Roberto Beck.
}

1) MD PhD. Department of Otolaryngology, University of São Paulo School of Medicine.

2) MD PhD. Department of Otolaryngology, University of São Paulo School of Medicine.

3) $\mathrm{MD} \mathrm{PhD}$. Heart Institute [InCor], University of São Paulo School of Medicine.

4) MD PhD. Department of Otolaryngology, University of São Paulo School of Medicine.

5) MD. Department of Otolaryngology, University of São Paulo School of Medicine.

Institutions: (A) Department of Otolaryngology, University of São Paulo School of Medicine.

(B) Heart Institute (InCor), University of São Paulo School of Medicine.

São Paulo/SP - Brazil.

Mailing address: Jeanne Oiticica, M.D., Ph.D. ENT Assistant Doctor Department of Otolaryngology University of São Paulo School of Medicine - R. Marjorie Prado 160 - São Paulo / SP - Brazil - Zip Code: 04663-080 - E-mail: jeanneoiticica@bioear.com.br

Article received on February $27^{\text {th }}, 2013$. Article accepted on March $17^{\text {th }}, 2013$

\section{SUMMARY}

Introduction: Sudden hearing loss (SHL) is an ENT emergency defined as sensorineural hearing loss (SNHL) $\geq 30 \mathrm{~dB} \mathrm{HL}$ affecting at least 3 consecutive tonal frequencies, showing a sudden onset, and occurring within 3 days. In cases of SHL, a detailed investigation should be performed in order to determine the etiology and provide the best treatment. Otoacoustic emission (OAE) analysis, electronystagmography (ENG), bithermal caloric test (BCT), and vestibular evoked myogenic potential (VEMP) assessments may be used in addition to a number of auxiliary methods to determine the topographic diagnosis.

Objective: To evaluate the contribution of OAE analysis, BCT, VEMP assessment, and magnetic resonance imaging (MRI) to the topographic diagnosis of SHL.

Method: Cross-sectional and retrospective studies of 21 patients with SHL, as defined above, were performed. The patients underwent the following exams: audiometry, tympanometry, OAE analysis, BCT, VEMP assessment, and MRI. Sex, affected side, degree of hearing loss, and cochleovestibular test results were described and correlated with MRI findings. Student's t-test was used for analysis of qualitative variables $(\mathrm{p}<0.05)$.

Results: The mean age of the 21 patients assessed was $52.5 \pm 15.3$ years; $13(61.9 \%)$ were women and 8 (38.1\%) were men. Most (55\%) had severe hearing loss. MRI changes were found in $20 \%$ of the cases. When the audiovestibular test results were added to the MRI findings, the topographic SHL diagnosis rate increased from $20 \%$ to $45 \%$.

Conclusion: Only combined analysis via several examinations provides a precise topographic diagnosis. Isolated data do not provide sufficient evidence to establish the extent of involvement and, hence, a possible etiology.

Keywords: Deafness; Hearing Loss, Sudden; Diagnosis; Vestibular Function Tests; Hearing Tests.

\section{INTRODUCTION}

Sudden hearing loss (SHL) is an ENT emergency that was first described in 1944 (1), but remains poorly understood. According to the National Institute for Deafness and Communication Disorders (NIDCD), it can be defined as any sensorineural hearing loss (SNHL) $\geq 30 \mathrm{~dB}$ affecting at least 3 consecutive frequencies, showing a sudden onset, and occurring within 3 days (2).

The incidence of SHL is 5-20 per 100,000 population per year in the United States (3), 8 per 100,000 population per year in Thailand (4), and 27.5 per 100,000 population per year in Japan (5), where a increase has been observed over the past 30 years. The natural course leads to spontaneous recovery in $45-65 \%$ of the cases (6-8).
Among patients undergoing drug therapy, recovery rates vary between $50 \%$ and $78 \%(5,9)$. About half of the patients undergoing treatment recover hearing within the first few days and the other half within 3 months, but a small percentage show delayed recovery (9).

Despite its sudden onset, which is often associated with symptoms such as dizziness and tinnitus, the pathophysiology of this condition remains undefined. Routine tests for diagnostic evaluation fail to detect the etiology in up to $88 \%$ of the cases (10). The list of potentially causative or associated agents is long; many of these are etiologic factors such as vestibular nerve schwannoma, infections, stroke, and neoplastic lesions, whereas others are only associated factors for which a causal relationship remains to be established (viral, autoimmune, vascular mechanisms) (11-13). Early diagnosis 
and therapeutic management improve the patients' quality of life.

Tinnitus and aural fullness are reported in $95 \%$ of the patients with SHL, whereas dizziness is present in 55\% of the cases showing the involvement of the posterior labyrinth and suggesting a worse prognosis (14). A detailed investigation of patients with SHL should be performed in all cases, the main objective of which should be to determine the etiology of the event in an effort to provide the best treatment for that patient. Furthermore, clinical evaluation may provide additional new data and a more detailed overview of the prognosis of patients with idiopathic SHL.

Recent studies have demonstrated the role of tests such as otoacoustic emission (OAE) analysis, electronystagmography (ENG), bithermal caloric test (BCT), and vestibular evoked myogenic potential (VEMP) assessment in indicating disease prognosis, and also elucidated various auxiliary methods for determining the topographic diagnosis (15-17).

OAE analysis is an important test for objective assessment of the functioning of the inner ear, specifically the outer hair cells (OHCs) of the cochlea. OAEs are lowintensity acoustic signals generated by nonlinear mechanical activity of the OHCs of the organ of Corti (18). OAEs can occur spontaneously (spontaneous OAEs) or in response to acoustic stimuli (evoked OAEs), which, once amplified, can be detected in the external auditory canal (EAC) (19). The sound stimulus reaches the cochlea and induces vibration of the basilar membrane, which in turn causes deflection of OHC stereocilia, ion flow, a voltage difference, and subsequent contraction of these cells to generate electromotility, which is thought to be responsible for the phenomenon of OAEs. The presence of OAEs indicates that the conductive mechanisms of the ear (external ear canal, tympanic membrane, and ossicular chain) and the OHCs are functioning properly, and therefore, in any kind of hearing loss (HL), OAE assessment is a valuable step. However, we must remember that OAEs do not provide information about the inner hair cells (IHC), the eighth cranial nerve, or the ascending auditory pathways. Spontaneous OAEs reveal narrow band acoustic energy from the cochlea regardless of the presence of a sound stimulus. They are of little clinical importance because they are present in only $40-60 \%$ of subjects with normal hearing (20). Evoked OAEs can be classified as transient-evoked otoacoustic emissions (TEOAEs) and distortion-product otoacoustic emissions (DPOAEs). TEOAE responses are caused by acoustic stimuli, usually clicks, but frequencyspecific stimuli such as tone bursts and tone pips can also induce these responses. Although a click is a broadband stimulus that activates the whole cochlea, TEOAEs can provide the "frequency-specific" pattern of the cochlea. They can be divided into frequency bands representing responses from different segments of the cochlea (21). DPOAEs are generated in the cochlea in response to the simultaneous presentation of 2 pure tones ( $\mathrm{f} 1$ and $\mathrm{f} 2$ stimuli). The cochlea has nonlinear properties that produce changes in the output signal that are not directly related to the input signal, creating responses at frequencies other than those provided by the 2 input signals. These responses are called distortion products and indicate normal activity of the inner ear. The $2 \mathrm{f} 1-\mathrm{f} 2$ ratio is the most commonly used as it results in the most robust and reliable responses. DPOAEs are present in almost all subjects with normal hearing thresholds. OAEs have been widely used as an objective and non-invasive screening method for HL. OAEs can still be useful in the differential diagnosis of cochlear and retrocochlear HL. As they arise from OHCs (peripheral auditory system), responses are supposed to be compatible with auditory thresholds in the case of sensory HL. In the case of retrocochlear pathology, DPOAE responses may be present even with thresholds worse than $45 \mathrm{~dB}$ HL and abnormal ABR since DPOAEs reflect pre-neural sensory activity from the cochlea. However, this is an uncommon finding. Studies indicate that about $20 \%$ of patients with a retrocochlear pathology have normal DPOAEs. Expanding lesions in the internal auditory canal (IAC) or posterior fossa may decrease the cochlea blood flow and affect the presence of OAEs. In SHL, the OAE test is a fast and elegant method for confirming HL, excluding any psychogenic deafness, and monitoring treatment outcome (17).

The ENG permits electrical recording of eye movements and is the most common method used for the diagnosis of peripheral vestibular disorders. The caloric test allows for independent assessment of the right and left labyrinths (horizontal semicircular canals) in response to bithermal irrigation of the external auditory canal. Each ear is irrigated twice to elicit both excitatory and inhibitory responses. Thus, the caloric test provides a functional assessment of the horizontal semicircular canal and superior vestibular nerve, contributing to the topographic study of the peripheral organ and ascending vestibular pathways.

The VEMP assessment is a newly developed vestibular test that is used clinically to analyze otolith function and is the only specific test for the inferior vestibular nerve. In primitive vertebrates, the saccule is not part of the vestibular system but is a structure of the auditory system. In most vertebrates and humans, the saccule is sensitive to acoustic stimuli but is involved with vestibular function. Furthermore, as a noninvasive and low-risk test, the VEMP assessment does not depend on cochlear integrity and may be present in patients with profound deafness. This potential can be evoked by a sound stimulus and recorded in the cervical region. In this way, VEMP analysis assesses the 
integrity of the sacculocollic reflex, which depends on the functional integrity of the macula of the saccule, inferior vestibular nerve, lateral vestibular nucleus, descending vestibulospinal tract, and the accessory nerve to the sternocleidomastoid muscle (SCM) and its neuromuscular junction. Therefore, unlike other exams, VEMP assessment enables the study of structures not commonly assessed by traditional vestibular tests (the saccule and inferior vestibular nerve) as well as the descending and ascending vestibular pathways. Lesions anywhere in this pathway can result in abnormal test results. The literature on VEMP in patients with SHL remains very controversial. Some authors describe a positive correlation between the presence of normal VEMP and a good prognosis for hearing recovery in patients with SHL $(22,23)$. Other authors have noted normal VEMP results in all patients with SHL (24), or did not relate these to hearing outcome. However, VEMP assessment can contribute to the diagnosis of different neurotologic diseases, including Meniere's disease, superior semicircular canal dehiscence, benign paroxysmal positional vertigo (BPPV), vestibular neuritis, and vestibular schwannoma, and it is a great method for topographic diagnosis. It evaluates not only the neural structures, but also the sensory structures of the saccule, which are sensitive and responsive to acoustic stimuli, although they do not contribute to hearing. For example, in a case of SHL of vascular origin, the lesion is expected to be large and involve not only the cochlea, but also the vestibule, damaging a greater number of sensory-neural structures. In such cases, combined analysis of audiological and electrophysiological test results and imaging studies may contribute directly to the topographic diagnosis of the lesion and to the subsequent prognosis of the patient's SHL. Currently, the role of these diagnostic methods in the field of SHL remains uncertain, and more studies are needed to better determine the practical implications of these tests.

\section{OBjeCtIVE}

To evaluate the contribution of OAE analyses, the bithermal caloric test, VEMP assessments, and magnetic resonance imaging (MRI) to the topographic diagnosis of SHL.

\section{Method}

This cross-sectional study included patients with a history of SHL, as defined above, who consulted the Department of Neurotology, Hospital das Clínicas, University of São Paulo School of Medicine (HC-FMUSP) from January 2011 to January 2012. This study was previously approved by the Hospital's Ethics Committee on Research (1179/
07). All patients followed the outpatient care protocol for SHL, including providing a detailed history, and undergoing an ENT physical examination as well as the following exams: pure tone and speech audiometry, tympanometry, DPOAE analysis, ENG, VEMP assessment, laboratory tests, and MRI. All tests were performed at the University of São Paulo School of Medicine.

\section{Inclusion criteria}

We included all patients who satisfied the following criteria:

- Were diagnosed with SHL (sensorineural hearing loss $\geq$ $30 \mathrm{~dB}$ over at least 3 contiguous frequencies developed within 72 hours) and confirmed by pure tone audiometry; for patients who had no prior audiometry results, the audiometry findings of the contralateral side were considered representative of the original auditory threshold.

- Performed the DPOAE test.

\section{Exclusion criteria}

We excluded patients who met any of the following criteria:

- Did not agree to participate in the study

- Failed to comply with the follow-up procedure or who had contraindications for any test (metallic objects implanted in the body preventing the realization of MRI, tympanic membrane perforation preventing the completion of ENG with water, middle ear effusion, otosclerosis or ossicular chain disjunction preventing DPOAE or VEMP assessments, as these conditions would interfere with the outcome).

\section{Pure tone and speech audiometry, tympanometry}

Air-and bone-conducted pure tone thresholds were obtained in the frequency range of 250 to $8000 \mathrm{~Hz}$ and 500 to $4000 \mathrm{~Hz}$, respectively, in addition to speech recognition threshold (SRT) and word recognition scores (WRS). When the patient showed no speech discrimination at the highest intensity permitted by the equipment, we tested the speech detection threshold (SDT). Immitance measurements included tympanometry and contralateral acoustic reflex thresholds at 500 to $4000 \mathrm{~Hz}$. The reflex was considered present when detected for at least 1 of 4 frequencies and absent when no response was detected for any frequency. The PTA (Pure Tone Average) was obtained by averaging the pure tone thresholds of $0.5,1$, 2, 4, 6, and $8 \mathrm{kHz}$. 


\section{Otoacoustic emissions - OAE}

Before the OAE test, an otoscopy was performed to prevent cerumen or debris from blocking the probe (21). To ensure proper acquisition of OAEs, the test was performed in a quiet environment with a noise level $\leq 40 \mathrm{~dB}$ HL. The patient was instructed to relax and avoid jaw movements, swallowing, or voice emissions that could generate noise in the external auditory canal (21). DPOAEs were tested at the intensities of $65 \mathrm{~dB}$ HL (f1) and $55 \mathrm{~dB}$ $\mathrm{HL}$ (f2) in the frequency range 750 to $8000 \mathrm{~Hz}$. A response was considered to be significant when the signal-to-noise ratio was $\geq 6 \mathrm{~dB}$. When significant responses were obtained for 5 or more frequencies, DPOAEs were considered to be present. In this case, further analysis was undertaken to determine whether DPOAEs were consistent with auditory thresholds. Present responses showed proper cochlear function of OHCs at these frequencies. We used SCOUT software (Natus Medical Incorporated, Mundelein, Il, USA) for these analyses.

\section{Bithermal Caloric Test - BCT}

Before the BCT analysis, the patient underwent a standard ENG test battery with the aim of excluding other functional findings that could influence the post-caloric response. The test sequence consisted of recordings of eye movements in response to stimuli: (1) saccade test, (2) spontaneous nystagmus with open eyes (SNEO), (3) smooth pursuit (SP), (4) optokinetic test (OPK), (5) static position tests - supine, right ear down, left ear down, Rose, sitting, body right and body left, (6) BCT. For the BCT, the patient was in a supine position with the head bent forward $30^{\circ}$ and eye movements were recorded after irrigation with water for 40 seconds in the following sequence: (a) warm irrigation at $44^{\circ} \mathrm{C}$, left ear, (b) warm irrigation at $44^{\circ} \mathrm{C}$, right ear, (c) cool irrigation at $30^{\circ} \mathrm{C}$, left ear, (d) cool irrigation at $30^{\circ} \mathrm{C}$, right ear. In the BCT analysis, we considered nystagmus of both labyrinths, measured through the angular velocity of the slow-phase velocities (SPV). We observed the nystagmus direction, rhythm, amplitude, and frequency, and compared the functioning of the labyrinths. The SPV measure was provided by the computer system and was considered normal between 7 and $50 \%$ second. The results were classified according to the post-caloric values: (1) normal: post-caloric responses within these limits, (2) hyperactivity: post-caloric responses exceeding $50 \%$ second, (3) hypoactivity: post-caloric responses below $7 \%$ second, and (4) no response: complete absence of caloric responses after stimulation. To evaluate the response symmetry between the vestibules, 2 values were calculated: (a) Unilateral Weakness (UW) and (b) Directional Preponderance (DP). UW shows the relative difference between the right and left ear responses, and DP shows the relative difference of right beating vs. left beating nystagmus directions. We considered UW up to $18 \%$ and DP up to $20 \%$ as reference values (25). UW has a high clinical value and indicates a vestibular asymmetry of peripheral or central origin; it always points to the weaker ear. DP indicates the stronger nystagmus direction. We considered signs of central origin to include the absence of the inhibitory effect of ocular fixation suppression (FS) and reversal of the direction of post-caloric nystagmus.

\section{Vestibular evoked myogenic potentials - VEMP}

A cervical VEMP assessment was performed using AEP software, version 7.0.0, Bio-logic Navigator Pro system (Natus Medical Incorporated, Mundelein, Il, USA). During the test, the patient remained comfortably seated with neck rotation contralateral to the sound stimulation. The sound stimuli were presented through insert earphones (ER-3) calibrated according to ANSI S1.40-1984 (American National Standard Institute, 2001). The surface electrodes were placed on the forehead (ground), the upper part of the sternum (negative), and the upper third of the contracted SCM muscle (positive electrode). The impedance was $\leq 5$ kilohms (KÙ). We used monoaural stimulation with $500 \mathrm{~Hz}$ tone bursts (rarefaction polarity) delivered at a rate of 4.3/ sec, beginning at $95 \mathrm{~dB} \mathrm{HL}$ with a $10 \mathrm{~dB}$ down-seeking procedure to determine the VEMP threshold. At each run, 150 stimuli were presented. The recordings were repeated to ensure reliable responses. Patients were allowed rest periods between each run to prevent cervical muscle fatigue. Each side was tested separately. We assessed the presence or absence of responses, latencies (P1 around 13 ms and $\mathrm{N} 1$ around $23 \mathrm{~ms}$ ), amplitudes of P1 and N1, as well as response thresholds and the interaural amplitude difference ratio (IADR). The formula was as follows: IADR (\%) = (greater amplitude side - smaller amplitude side / greater amplitude side + smaller amplitude side) X 100. Qualitative (presence or absence of responses) and quantitative (latencies and IADR) analyses were performed to compare the values of the ear with SHL and the contralateral ear. For amplitude measurements and IADR, the $95 \mathrm{~dB} \mathrm{HL}$ recording with the largest amplitude in each ear was selected. As abnormal VEMP response, we considered an IADR $>40 \%$ (with the lowest amplitude from the SHL side) or the absence of a response on the affected side, suggesting saccule and inferior vestibular nerve involvement.

\section{Magnetic resonance imaging (MRI) of the inner ear and brain stem}

MRI of the inner ear was carried out by spin-echo and fast spin-echo sequences in T1 and T2, with multiplanar 
acquisition before and after intravenous administration of the paramagnetic contrast agent (gadolinium). FIESTA series were also included in volume acquisition with cuts of $0.8 \mathrm{~mm}$. MRI was considered abnormal only when findings were obviously related to SHL, such as schwannoma of the eighth cranial nerve (intracanalicular or cerebellopontine angle), internal carotid artery obstruction, stroke, and cochleovestibular lesions (inflammation or vascular), as previously described (26). All other MRI findings were described as normal, including those whose etiological association with SHL were possible but not previously proven (vascular anomalies, demyelinating diseases) or unknown (white matter changes, vascular loops, contralateral cochlear abnormalities, enhancement of mastoid cells or the endolymphatic duct, ventricular dilatation, asymmetry of the cerebellum).

\section{Study variables}

Study variables included the following: (a) categorical variables (CVs), and (b) quantitative variables (QVs). The CVs were described by their frequency distribution, and included the following variables: (1) sex; (2) the affected side; (3) classification of the degree of HL according to pure tone thresholds (up to $40 \mathrm{~dB} \mathrm{HL}$ mild, 40-70 dB HL moderate, 70-90 dB HL severe, and $>90 \mathrm{~dB}$ HL profound) (27). We considered anacusis as the complete absence of responses at all frequencies; (4) The presence or absence of the acoustic reflex during immitance measurements; (5) the presence or absence of DPOAE responses; (6) the presence of normal reflexes, hyperfunction, hypofunction, or no responses in the BCT; (7) normal or abnormal VEMP responses; and (8) normal and abnormal MRI findings. The QV were described by mean and standard deviation, and included (1) age; (2) PTA; (3) WRS.

\section{Statistical analysis}

CV results were described based on their frequency. QV results were expressed as the mean and standard deviation. Comparisons of QV were calculated using an unpaired t-test. The level of statistical significance was set at $5 \%(\mathrm{p}<0.05)$. We also calculated the $95 \%$ confidence interval of the mean and verified whether the $2 \mathrm{QV}$ had equal standard deviations. Statistical analyses were performed using the GraphPadInstat program.

\section{RESULTS}

Between January 2011 and January 2012, 40 patients diagnosed with SHL were treated at the Department of Neurotology, Hospital das Clínicas, University of São Paulo

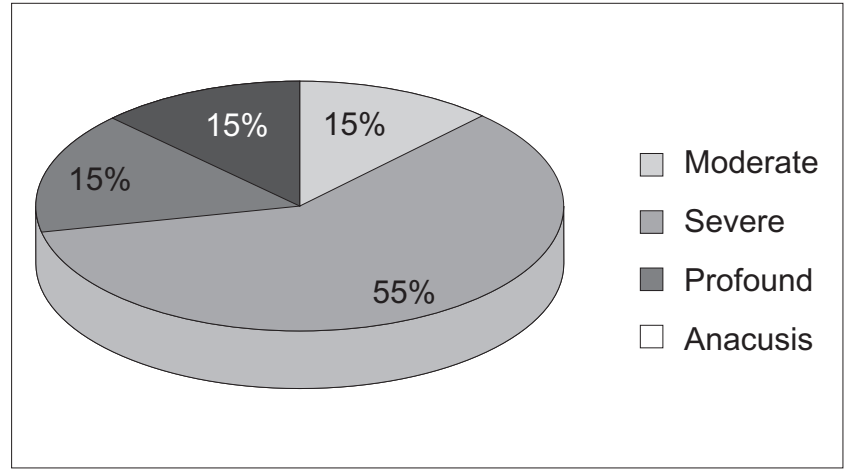

Figure 1. Classification of NSHL in patients with SHL.

School of Medicine. Twenty-one of these had DPOAEs and constituted our final sample. No patient refused to participate in the study, had contraindications to any of the exams, or missed their follow-up visits. The analysis of the 21 patients who met the selection criteria showed that the mean age and standard deviation was $52.5 \pm 15.3$ years, and that 13 (61.9\%) were females and 8 (38.1\%) males. The right side was affected in $14(66.6 \%)$ patients and the left side in 7 (33.4\%).

\section{Pure tone and speech audiometry, tympanometry}

Of the 21 enrolled patients, 20 underwent audiometry at the beginning and end of the study, only 1 patient only underwent the initial audiometry tests. Figure 1 shows the distribution of the degree of NSHL observed in 20 patients at the beginning of the analysis. The initial mean PTA and standard deviation was $80.5 \pm 25.7 \mathrm{~dB} \mathrm{HL}(\mathrm{n}=20)$, with a $95 \%$ confidence interval of the mean between 68.5 and $92.6 \mathrm{~dB}$. The mean PTA at the end of the study and the standard deviation was $57.6 \pm 34.3 \mathrm{~dB} \mathrm{HL}(\mathrm{n}=20)$, with a $95 \%$ confidence interval of the mean between 41.5 and $73.7 \mathrm{~dB}$. The difference between the initial and final PTA was statistically significant ( $p=0.0221$, unpaired t-test), indicating that, in general, there was an improvement in the thresholds during follow-up due to treatment of SHL.

\section{ENG and PC}

Nineteen of the 21 patients (90.5\%) had ENG with caloric tests. Two (9.5\%) failed to show up for the test. Of the 19 patients on whom the tests were performed, 8 (42.1\%) had normal and 11 (57.9\%) abnormal findings. One subject had DP on the same side as the SHL. The distribution of the remaining 18 test results of the affected ears can be seen in Figure 2 . 


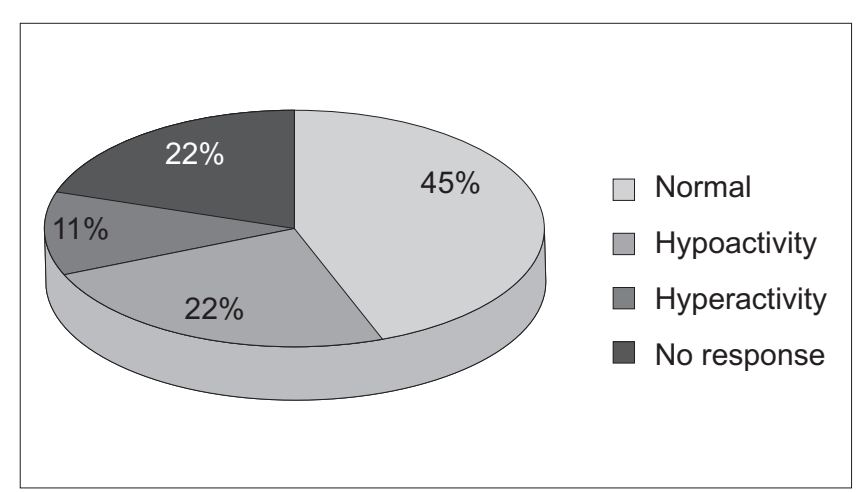

Figure 2. Distribution of bithermal caloric test responses in patients with SHL.

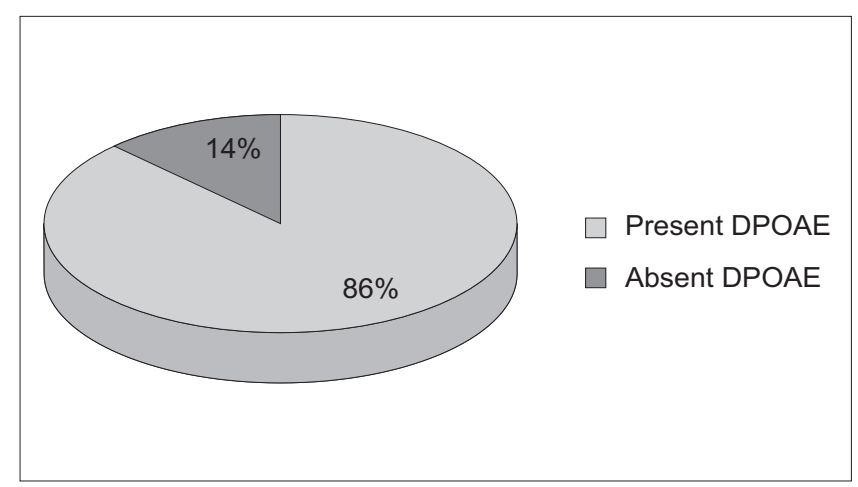

Figure 4. Distribution of DPOAE results in patients with SHL.

Fourteen of 21 patients (66.6\%) completed the VEMP test. Nine of these (64.3\%) had normal tests. Of the $5(35.7 \%)$ with abnormal test results, 3 had an IADR $>40 \%$, with the lowest amplitude response on the SHL side in all cases; 2 had no responses on the affected side (Figure 3).

\section{DPOAE}

All 21 patients included in the study completed DPOAE assessments. Only 3 (14.3\%) showed present DPOAEs at most frequencies. Although DPOAE were requested at the time of initial evaluation, in view of the test schedules and the demand of the institution, some of these tests were eventually completed during follow-up of SHL after partial or complete recovery of NSHL, and the results were consistent with the audiometric thresholds obtained at the same time as the DPOAE tests. In the remaining 18 (85.7\%) patients, DPOAEs were considered absent according to our criteria (Figure 4).

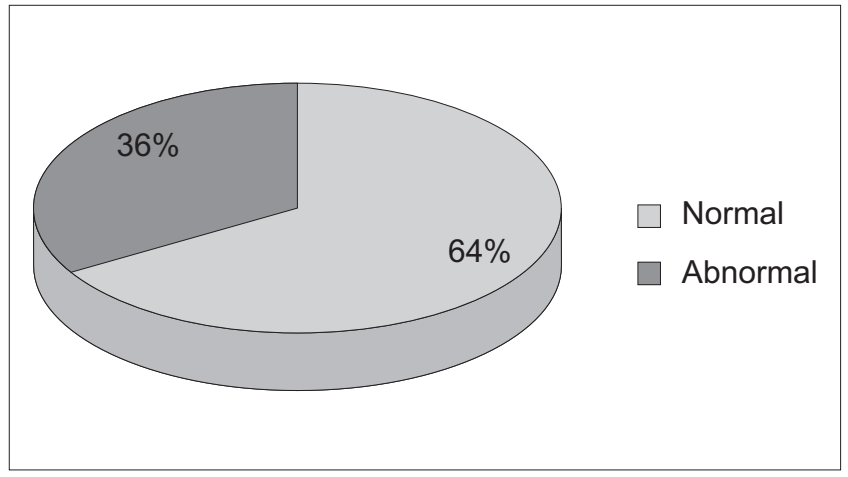

Figure 3. Distribution of VEMP responses in patients with SHL.

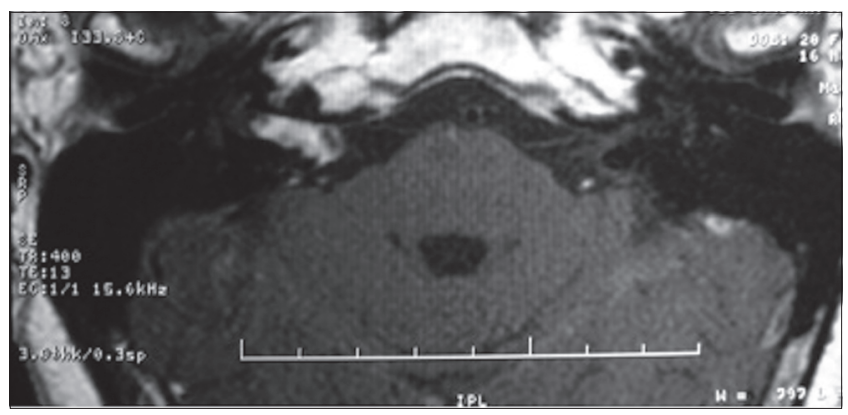

Figure 5. Expansive lesion inside the right IAC.

MRI was performed in 20 (95.2\%) of 21 patients as 1 patient failed to complete the test. The MRI findings were normal in 16 (80\%) patients and abnormal in 4 (20\%). Analyzing the 4 abnormal tests, we found that in 2 cases the lesion arose from the eighth cranial nerve (schwannoma) ipsilateral to the SHL. In case 9, MRI revealed an expansive lesion inside the right IAC, protruding into the ipsilateral cerebellopontine angle (CPA) (Figure 5). In case 11, MRI showed an expansive lesion located in the right CPA and IAC, with enlargement and partial obliteration of the ipsilateral cistern (Figure 6). Among the 2 patients with eighth cranial nerve schwannomas, DPOAEs were absent in 1 (case 9) but present in the other (case 11). In this case, the test was performed later, during follow-up of SHL, and after complete recovery of audiometric hearing thresholds. Similarly, VEMP was absent in case 9 and present in case 11. ENG showed hypofunction in case 9 and no caloric function at all in case 11 . In the other 2 cases with abnormal MRIs, the lesion was located in the cochlea on the same side as the SHL. The lesion was described by the radiologist 
as a focus of post-contrast enhancement in the cochlea, vestibule, and ipsilateral semicircular canals suggestive of inflammation (viral labyrinthitis, fibrotic tissue) in 1 (case 6), and as enhancement of the ipsilateral cochlea (possible inflammation) in the other (case 18). In the 2 latter cases, DPOAEs were absent, which was in agreement with the MRI findings showing cochlear enhancement, and helped to prove that the auditory lesion was peripheral, involving the OHCs, and not neural. VEMP was abnormal in case 6 and was not assessed in case 18. Thus, ENG showed no vestibular function ipsilateral to the lesion in both cases, even without any sign on MRI of a lesion extending into the posterior labyrinth in case 18 .

We also evaluated the mean difference between the initial and final PTA to compare the average hearing recovery among patients whose topographic diagnosis showed smaller, more restricted lesions (absent or Cn or $\mathrm{SVn}$ lesions) or more extensive lesions ( $\mathrm{Cn}+\mathrm{VSn}$ or $\mathrm{Cn}+$ $\mathrm{VSn}+\mathrm{IVn})$. The mean PTA recovery and standard deviation

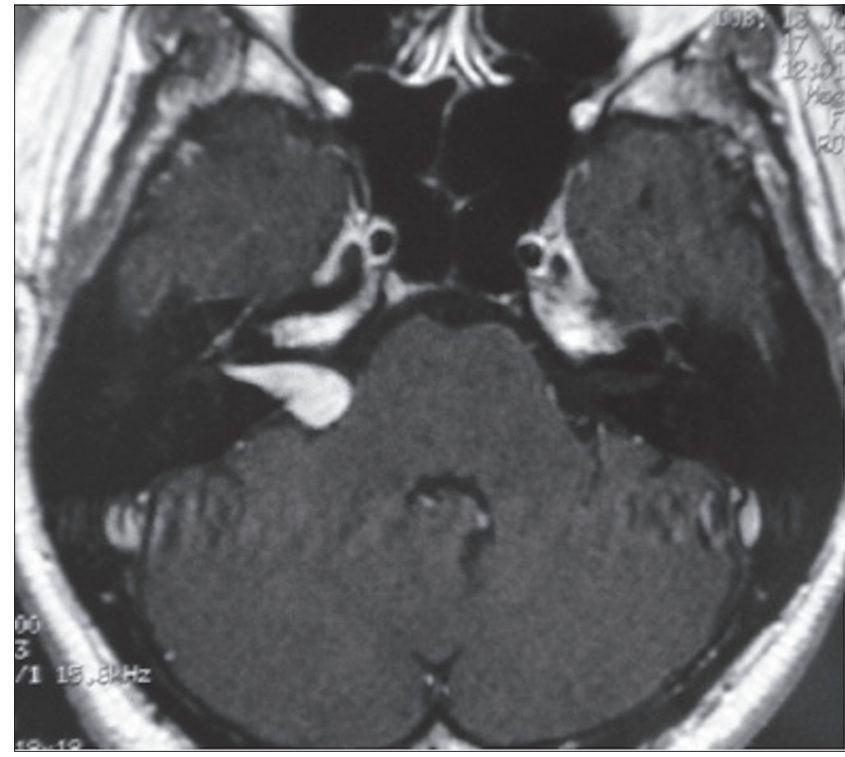

Figure 6. Expansive lesion in the right IAC and CPA with widening and partial obliteration of the ipsilateral CPA cistern.

Table I. Test results for all 2 I subjects.

\begin{tabular}{|c|c|c|c|c|c|c|c|c|c|}
\hline Case & Side & $\begin{array}{l}\text { Audio } \\
\text { InitialPTA } \\
(\mathrm{dBHL})\end{array}$ & $\begin{array}{l}\text { metry } \\
\text { FinalPTA } \\
(\mathrm{dBHL})\end{array}$ & I & DPOAE & $\mathrm{BCT}$ & VEMP & $\mathrm{MRI}$ & $\begin{array}{l}\text { Topographic Diagnosis } \\
\text { (affected nerve) }\end{array}$ \\
\hline | & $\mathrm{R}$ & 75.8 & 73.3 & NP & - & h & NP & $\mathrm{nl}$ & $\mathrm{Cn}_{\mathrm{n}}$ \\
\hline 2 & $\mathrm{~L}$ & 80.8 & 25 & + & - & $\mathrm{nl}$ & $\mathrm{nl}$ & $\mathrm{nl}$ & Cn \\
\hline 3 & $R$ & 50.8 & 24 & - & + & - & $\mathrm{nl}$ & $\mathrm{nl}$ & $S V_{n}$ \\
\hline 4 & $\mathrm{~L}$ & 59 & NP & + & - & NP & NP & $\mathrm{nl}$ & Cn \\
\hline 5 & $\mathrm{R}$ & 86.7 & 79 & NP & - & - & Abn & $\mathrm{nl}$ & $\mathrm{Cn}+\mathrm{SVn}+\mathrm{IVn}$ \\
\hline 6 & $\mathrm{R}$ & 47.5 & 29 & NP & - & 0 & Abn & Abn* & $\mathrm{Cn}+\mathrm{SVn}+\mathrm{IVn}$ \\
\hline 7 & $\mathrm{R}$ & 81.6 & 25 & NP & + & $\mathrm{nl}$ & $\mathrm{nl}$ & $\mathrm{nl}$ & - \\
\hline 8 & $\mathrm{~L}$ & 120 & 95,8 & NP & - & 0 & Abn & $\mathrm{nl}$ & $\mathrm{Cn}+\mathrm{SVn}+\mathrm{IVn}$ \\
\hline 9 & $\mathrm{R}$ & 120 & 120 & NP & - & - & Abn & Abn料 & $\mathrm{Cn}+\mathrm{SVn}+\mathrm{IVn}$ \\
\hline 10 & $\mathrm{R}$ & 64 & 35 & NP & - & - & $\mathrm{nl}$ & NP & $C n+S V n$ \\
\hline | | & $\mathrm{R}$ & 37.5 & 10 & & + & 0 & $\mathrm{nl}$ & Abn ***** & SVn \\
\hline 12 & $\mathrm{R}$ & 70,8 & 64,1 & NP & - & $\mathrm{nl}$ & $\mathrm{nl}$ & $\mathrm{nl}$ & $\mathrm{Cn}$ \\
\hline 13 & $\mathrm{R}$ & 69 & 25,8 & + & - & $\mathrm{nl}$ & NP & $\mathrm{nl}$ & Cn \\
\hline 14 & $\mathrm{R}$ & 65 & 46 & NP & - & h & $\mathrm{nl}$ & $\mathrm{nl}$ & $\mathrm{Cn}+\mathrm{SVn}$ \\
\hline 15 & $L$ & 67.5 & 81 & - & - & $\mathrm{nl}$ & NP & $\mathrm{nl}$ & Cn \\
\hline 16 & $\mathrm{R}$ & 81.6 & 22,5 & - & - & $\mathrm{nl}$ & NP & $\mathrm{nl}$ & $\mathrm{Cn}$ \\
\hline 17 & $L$ & | 17.5 & 120 & NP & - & $\mathrm{DP}$ & Abn & $\mathrm{nl}$ & $\mathrm{Cn}+\mathrm{SVn}+\mathrm{IVn}$ \\
\hline 18 & $\mathrm{~L}$ & 120 & 120 & NP & - & 0 & $N R$ & Abn粎米 & $C_{n}+S V_{n}$ \\
\hline 19 & $L$ & 83.3 & 47.5 & NP & - & $\mathrm{nl}$ & $\mathrm{nl}$ & $\mathrm{nl}$ & Cn \\
\hline 20 & $\mathrm{R}$ & 120 & 76.6 & NP & - & $N R$ & $N R$ & $\mathrm{nl}$ & Cn \\
\hline 21 & $\mathrm{R}$ & 112.5 & 104 & NP & - & $\mathrm{nl}$ & $\mathrm{nl}$ & $\mathrm{nl}$ & Cn \\
\hline
\end{tabular}

I: immitance measurements; DPOAE: distortion-productotoacousticemissions; BCT: bithermal calorictest; VEMP: vestibularevoked myogenic potential; MRI: magnetic resonance imaging; R: Right, L: left; dB: decibels; -: absent, + : present; $\uparrow$ : hyperactivity; $\downarrow$ : hypoactivity; nl: normal; 0: no response, DP: directional preponderance; NP: not performed; Cn: cochlear nerve; SVn: superiorvestibular nerve; IVn: inferior vestibular nerve; Abn: Abnormal, -: none; *: Spotlights of post-contrastenhancement in the cochlea, vestibule, and right HSC that could represent inflammation (viral labyrinthitis, fibrotic tissue); ***: expansive lesion within the right internal auditory canal protruding into the ipsilateral cerebellopontine angle; ****: expansive lesion in the right internal auditory canal and cerebellopontine angle with widening and partial obliteration of the ipsilateral cistern; *****: area of enhancement in the left cochlea (inflammation?). 
was $29.3 \pm 23.8 \mathrm{~dB}(\mathrm{n}=12)$, with a $95 \%$ confidence interval of the mean between 14.1 and $44.5 \mathrm{~dB}$ in patients with topographically more restricted lesions. In patients with topographically more extensive lesions the mean PTA recovery and standard deviation was $13.2 \pm 10.9 \mathrm{~dB}(\mathrm{n}=$ 8) with a $95 \%$ confidence interval of the mean between 4 and $22.4 \mathrm{~dB}$. Case 4 was excluded from the analysis because only the initial audiometry results were available and no differences between the initial and final PTA could be calculated. The difference in the mean PTA recovery among patients with topographically more restricted lesions was statistically significantly different $(p=0.05$, unpaired t-test) from those with more extensive lesions, indicating that patients with more extensive lesions show poorer hearing recovery.

\section{DISCUSSION}

In this cross-sectional study of 21 patients with SHL, abnormal MRI findings were seen in 20\% of cases, which is similar to the results of a previous report on SHL at our institution that showed abnormal MRI findings in 25\% of those studied (28). The small difference in abnormal MRI findings may be due to the smaller sample size used in this study. We chose to use DPOAE as an inclusion criterion because the test permits the evaluation of each frequency separately and responses may be present with hearing thresholds up to $45 \mathrm{~dB} \mathrm{HL}$, whereas TEOAEs are usually absent when the thresholds exceed 20-30 dB HL (29). Therefore, this test is not only important for the differential diagnosis of peripheral and central lesions, but also because absent responses in the initial SHL evaluation exclude the possibility of psychogenic deafness (PD) or simulation.

Psychogenic deafness (PD) is a type of conversion disorder and can be defined as HL that cannot be explained by organic lesions or anatomical or physiological changes. In such cases, the patient's clinical history reveals psychological factors that could act as a trigger at the beginning of the event. It is possible to establish the diagnosis by comparing behavioral (pure tone audiometry) and objective tests (ABR and DPOAE), as these usually show divergent results: patients typically exhibit elevated pure tone thresholds but normal ABR and DPOAE responses. Attention is drawn to case 7 in Table 1. This patient presented with initial severe $\mathrm{HL}$ although tests failed to show any evidence of a structural lesion and follow-up showed a full recovery. One hypothesis for this case is PD. Malingering may also lead to divergent results between behavioral and objective tests, but in contrast to PD, it is usually is associated with an inconsistent history, personal advantages associated with deafness, and differences between pure tone audiometry and SRT $\geq 15 \mathrm{~dB}$, which can be detected by an experienced audiologist. Therefore, in routine clinical evaluation of patients with SHL, DPOAE is a valid tool and contributes to diagnostic accuracy. If an unexpected response is present during the initial evaluation, the possibility of a retrocochlear lesion should be considered and investigated.

In our study, DPOAEs were eventually performed late during follow-up of SHL and not as part of the initial test as planned. When performed during follow-up, it may be useful for showing possible cochlear recovery. This can happen even if the cause of SHL is an expansive lesion of the IAC or PCA, provided cochlear blood flow is preserved and cochlear integrity is ensured. In case 11, normal VEMP and DPOAE test results suggested normal saccular, inferior vestibular nerve, and cochlear (OHC) function. Pure tone and speech audiometry results were normal at the time the DPOAE was performed, indicating complete hearing recovery after initial moderate HL. We speculate that the initial hearing impairment caused by the expansive lesion extending from the IAC to CPA may have improved as a result of decreased edema resulting in a favorable treatment outcome. In the same case, the absence of a right vestibular response, as shown by BCT, suggests that only the superior vestibular nerve was affected.

In case 9, the lesion was primarily located in the right IAC (although protruding into the CPA), and the test results (complete deafness on PTA, absent DPOAEs, abnormal VEMP, hypoactivity in BCT) confirmed that the whole cochleo-vestibular system was affected on this side. Therefore, in our view, DPOAE analysis is an important test for SHL evaluation since it is the only one that assesses cochlear function.

Patients underwent a complete ENG evaluation in order to rule out other changes to vestibular-ocular function suggestive of central involvement. No abnormalities suggesting central dysfunction were found in our series. Therefore, we considered only the BCT results in our analysis. The importance of vestibular evaluation in SHL becomes clear when we look at the cases with abnormal MRIs. Cases 6, 9, 11, and 18 were found to have structural lesions by MRI and all had impaired superior vestibular nerve function, with absent vestibular responses in $3(75 \%)$ of the 4 cases. Only 1 case had residual function, but hypoactivity (SPV below $7 \%$ sec), highlighting the importance of BCT for SHL prognosis. Overall, 4 cases in our sample showed no response, 3 (75\%) had abnormal MRIs and 2 of them ( $50 \%$ ) tumors, and so absent caloric test responses require an imaging study. However, abnormal imaging findings do not predict permanent deafness, since cases 6 and 11 both experienced hearing recovery. Audiovestibular function tests and MRI are important tools that together provide us with a complete overview of the functional impairment and the sites involved in SHL, and 
they may also be helpful for establishing the hearing prognosis for these patients.

MRI demonstrated structural changes in $20 \%$ of the patients ( 4 cases: 3 with an extensive lesion and 1 with a restricted lesion). When the audiovestibular test results were combined with the MRI findings, the proportion with a topographic SHL diagnosis increased from $20 \%$ to $45 \%$.

\section{CONCLUSION}

Only combined analysis of several test results allows for a precise topographic diagnosis. Isolated test results do not provide sufficient data to establish the extent of SHL involvement and hence a possible etiology. The combination of all tests was more efficient for topographic diagnosis than just audiometry and MRI. Combined assessment permits a functional evaluation that cannot be obtained with MRI alone. Each test evaluates a different segment of the cochleo-vestibular system and permits an overview of the degree of functional or structural impairment. The auditory evoked potentials (ABRs) could be included in the audiovestibular test battery. ABR may be useful to rule out malingering and patients with psychogenic hearing loss, and may also be helpful in follow-up of SHL, especially in patients with schwannomas.

\section{REFERENCES}

1. DeKleyn A. Sudden complete or partial loss of function of the octavus system in apparently normal persons. Acta Otolaryngol (Stockh). 1944;32:407-29.

2. National_Institute_of_Health.Sudden deafness. National Institutes of Health. NIH publication 00-4757: Bethesda Md; 2008.

3. Byl FM, Jr. Sudden hearing loss: eight years' experience and suggested prognostic table. Laryngoscope. 1984 May;94(5 Pt 1):647-61.

4. Wu CS, Lin HC, Chao PZ. Sudden sensorineural hearing loss: evidence from Taiwan. Audiol Neurootol. 2006;11(3):151-6.

5. Teranishi M, Katayama N, Uchida Y, Tominaga M, Nakashima T. Thirty-year trends in sudden deafness from four nationwide epidemiological surveys in Japan. Acta Otolaryngol. 2007 Dec;127(12):1259-65.

6. Mattox DE, Simmons FB. Natural history of sudden sensorineural hearing loss. Ann Otol Rhinol Laryngol. 1977 Jul-Aug;86(4 Pt 1):463-80.
7. Wilson WR, Byl FM, Laird N. The efficacy of steroids in the treatment of idiopathic sudden hearing loss. A doubleblind clinical study. Arch Otolaryngol. 1980 Dec;106(12):772-6.

8. Cole RR, Jahrsdoerfer RA. Sudden hearing loss: an update. Am J Otol. 1988 May;9(3):211-5.

9. Yeo SW, Lee DH, Jun BC, Park SY, Park YS. Hearing outcome of sudden sensorineural hearing loss: long-term follow-up. Otolaryngol Head Neck Surg. 2007 Feb;136(2):221-4.

10. Fetterman BL, Luxford WM, Saunders JE. Sudden bilateral sensorineural hearing loss. Laryngoscope. 1996 Nov;106(11):1347-50.

11. Bittar RS, Oiticica J, Zerati FE, Bento RF. Sudden hearing loss: a ten-year outpatient experience. Int Tinnitus J. 2009;15(2):196-202.

12. Oiticica J, Bittar RS. Metabolic disorders prevalence in sudden deafness. Clinics (Sao Paulo). 2010;65(11):1149553.

13. Mendes-Correa MC, Bittar RS, Salmito N, Oiticica J. Pegylatedinterferon/ribavirin-associated sudden hearing loss in a patient with chronic hepatitis C in Brazil. Braz J Infect Dis. 2011 Jan-Feb;15(1):87-9.

14. Park HM, Jung SW, Rhee CK. Vestibular diagnosis as prognostic indicator in sudden hearing loss with vertigo. Acta Otolaryngol Suppl. 2001;545:80-3.

15. Iwasaki S, Takai Y, Ozeki H, Ito K, Karino S, Murofushi T. Extent of lesions in idiopathic sudden hearing loss with vertigo: study using click and galvanic vestibular evoked myogenic potentials. Arch Otolaryngol Head Neck Surg. 2005 Oct;131(10):857-62.

16. Junicho M, Fushiki H, Aso S, Watanabe Y. Prognostic value of initial electronystagmography findings in idiopathic sudden sensorineural hearing loss without vertigo. Otol Neurotol. 2008 Oct;29(7):905-9.

17. Mori T, Suzuki H, Hiraki N, Hashida K, Ohbuchi T, Katoh A, et al. Prediction of hearing outcomes by distortion product otoacoustic emissions in patients with idiopathic sudden sensorineural hearing loss. Auris Nasus Larynx. 2011 Oct;38(5):564-9.

18. Brownell WE. Outer hair cell electromotility and otoacoustic emissions. Ear Hear. 1990 Apr;11(2):82-92.

19. Kemp DT. Stimulated acoustic emissions from within 
the human auditory system. J Acoust Soc Am. 1978 Nov;64(5):1386-91.

20. Bonfils P. Spontaneous otoacoustic emissions: clinical interest. Laryngoscope. 1989 Jul;99(7 Pt 1):752-6.

21. Kemp DT. Otoacoustic emissions, their origin in cochlear function, and use. Br Med Bull. 2002;63:223-41.

22. Wang CT, Huang TW, Kuo SW, Cheng PW. Correlation between audiovestibular function tests and hearing outcomes in severe to profound sudden sensorineural hearing loss. Ear Hear. 2009 Feb;30(1):110-4.

23. Korres S, Stamatiou GA, Gkoritsa E, Riga M, Xenelis J. Prognosis of patients with idiopathic sudden hearing loss: role of vestibular assessment. J Laryngol Otol. 2011 Mar;125(3):251-7.

24. Wu CC, Young YH. Vestibular evoked myogenic potentials are intact after sudden deafness. Ear Hear. 2002 Jun;23(3):235-8.

25. Halmagyi GM, Curthoys IS. A clinical sign of canal paresis. Arch Neurol. 1988 Jul;45(7):737-9.
26. Aarnisalo AA, Suoranta H, YlikoskiJ. Magnetic resonance imaging findings in the auditory pathway of patients with sudden deafness. Otol Neurotol. 2004 May;25(3):245-9.

27. Hornsby BW, Johnson EE, Picou E. Effects of degree and configuration of hearing loss on the contribution of highand low-frequency speech information to bilateral speech understanding. Ear Hear. 2011 Sep-Oct;32(5):543-55.

28. Bittar RSM, Sanchez TG, Sperandio F, Kii M, Formigoni LG, Bento RF. Utilidade da Ressonancia Magnética no diagnóstico etiológico da surdez súbita. Arq Int Otorrinolaringol. 1999;3(4):164-70.

29. Lonsbury-Martin BL, Martin GK. The clinical utility of distortion-product otoacoustic emissions. Ear Hear. 1990 Apr;11(2):144-54.

30. Harris FP, Probst R. Reporting click-evoked and distortionproduct otoacoustic emission results with respect to the pure-tone audiogram. Ear Hear. 1991 Dec;12(6):399-405.

31. Velenovsky DS, Glattke TJ. The effect of noise bandwidth on the contralateral suppression of transient evoked otoacoustic emissions. Hear Res. 2002 Feb;164(1-2):39-48. 